\title{
PROPUESTA DE IMPLEMENTACIÓN DE UN SISTEMA DE GESTIÓN AMBIENTAL PARA CAMPUS UNIVERSITARIO
}

\section{Clemencia Camacho Delgado}

\section{RESUMEN}

Es indudable que las universidades realizan diversas actividades con implicaciones ambientales de amplio rango. Los aspectos ambientales negativos van desde la generación de residuos, incluidos aquellos peligrosos producidos en laboratorios y centros médicos, hasta altos consumos de recursos con graves consecuencias sobre el entorno urbano. Los aspectos positivos están relacionados con el desarrollo de una conciencia en los nuevos profesionales, la investigación, difusión y aplicación de resultados en diferentes áreas ambientales, en estrecha unidad con la comunidad local y global donde ejercen una influencia definitiva en la mejora de la calidad de vida.

En este estudio se hizo una revisión de los Sistemas de Gestión Ambiental SGA, ejecutados en centros de educación superior, y se buscó aquellos que cuentan con la validación internacional o certificación. Se compararon y analizaron para contrastarlos con los contenidos definidos en las normas ISO 14001: 2001 para así establecer los puntos congruentes y elementos disímiles entre los SGA universitarios y el contenido de la norma.

Los resultados arrojaron una gran diferencia en la estructura de los SGA aplicados por las diferentes universidades, pues hubo casos de ausencia de definiciones en puntos categóricos, como la no declaración de una política institucional ambiental o la falta de objetivos y metas claras de acuerdo con lo estipulado en la norma ISO 14001.

\section{PALABRAS CLAVE}

Sistema de Gestión Ambiental, Norma ISO.

\section{ABSTRACT}

With no doubt universities carry out several activities that involve a wide range of environmental implications. The negative environmental aspects go from the production of waste, including those created in laboratories and medical centres which are very dangerous, all the way to the high consumption of resources and impacts around the urban area. The positive aspects are related to the creation of conscience in the new professionals, research, diffusion and application of results in different environmental areas, and the universities close unity with the local and global community where they have a definite influence on the improvement of the quality of life.

This study made a review of the Environmental Management Systems (EMS) used in higher educational institutions, looking for those that have an international ratification or certification. These systems were compared and analyzed in order to contrast them with the contents defined in the ISO 14001:2001 regulations and in this way, establish the points that were fulfilled, as well as the elements that are not alike between the EMS from the universities and the content of the regulation.

The results show a vast difference in the structure of the SGA applied by the different universities. We can even find cases in which there are no definitions in categorical points such as the no declaration of environmental institutional policy or the lack of objectives and clear goals according to what the ISO 14001 regulation stipulates. 
La universidad juega un papel fundamental en el éxito de politicas ambientales y es un agente clave, junto a los actores políticosy económicos, en la garantía del desarrollo sostenible.

\section{INTRODUCCIÓN}

Desde comienzos de la década de los 70 la sociedad mundial manifestó una creciente preocupación por el deterioro del medio ambiente. Hitos como la Conferencia de Estocolmo efectuada en 1972, el Informe de la Comisión Brundtland publicado en 1987 o la Cumbre de Río de Janeiro efectuada a mediados de 1992, empezaron a generar una preocupación por la protección de los recursos naturales y el medio ambiente en diferentes sectores de la sociedad.

En el año 1996 la Organización Internacional de Normalización (ISO), con sede en Ginebra (Suiza), publicó un grupo de normas técnicas (ISO 14000), orientadas a la ejecución de un Sistema de Gestión Ambiental (SGA) y a la responsabilidad ambiental de los productos o servicios, aplicables a cualquier institución o empresa y que fueran aceptadas mundialmente.

La norma NTC-ISO 14001 "Sistemas de Administración Ambiental, Especificaciones con Guía para Uso" fue adoptada en Colombia en 1996, año desde el cual, empresas con sentido de responsabilidad social y ambiental iniciaron las tareas de diseño e implantación del SGA. A comienzos del año 2000 se hizo manifiesta la intención de implementar un

\section{RESEÑA DEAUTOR}

Clemencia Camacho Delgado, bióloga y MSC. de la Pontificia Universidad Javeriana, con especialización en Gestión Ambiental de la Universidad Externado de Colombia. Diplomada en Docencia Universitaria en el Politécnico Grancolombiano, y en Enfoques Pedagógicos Contemporáneos de la Fundación Alberto Merani y el Politécnico Grancolombiano. Actualmente dirige la carrera de Administración de Empresas Agropecuarias y el Proyecto Institucional de Desarrollo Sostenible en el Politécnico Grancolombiano.
SGA en algunos centros académicos de Bogotá.

De acuerdo con la Asociación de Universidades Líderes en un Futuro Sostenible, (Association of University Leaders for a Sustainable Future, ULSF) ${ }^{1}$, el concepto de "sostenibilidad" en las universidades implica que "todas sus actividades deben ser ambientalmente favorables, socialmente justas y económicamente viables. Estos conceptos deben englobar el currículum, la investigación y la formación de profesionales, para que ellos contribuyan a un ambiente perdurable y a una sociedad justa".

\section{DEFINICIÓN DEL PROBLEMA}

La universidad juega un papel fundamental en el éxito de políticas ambientales y es un agente clave, junto a los actores políticos y económicos, en la garantía del desarrollo sostenible.

Hay razones importantes para implementar SGA en las universidades. En primer lugar, son pequeñas sociedades donde confluyen muy diversas actividades que se desarrollan en un espacio definido como "campus" y prestan igualmente, muy diversos servicios para la comunidad universitaria la cual comprende estudiantes, profesionales, académicos, personal administrativo y de servicios.

Estas actividades tienen implicaciones ambientales de amplio rango, tanto positivas como negativas. Los aspectos ambientales negativos van desde el manejo de residuos, incluidos los peligrosos generados en los laboratorios y el centro médico, altos consumos de recursos como agua y 
energía e impactos en el entorno urbano donde se ubica la universidad, entre otros. Los aspectos positivos están relacionados con el desarrollo de una conciencia ambiental en la comunidad universitaria, la investigación en diferentes áreas ambientales, con el concurso de docentes especializados para ello; el compromiso de formar profesionales con una visión sostenible y la relación estrecha de estas instituciones con la comunidad local y global, atendiendo a las políticas nacionales y mundiales.

La certificación de las universidades bajo la norma ISO 14001 genera nuevos caminos para hacer de estas instituciones una muestra de responsabilidad con su misión en los procesos educativos del país.

\section{METODOLOGÍA}

Se realizó una revisión bibliográfica sobre los SGA implementados en centros de educación superior, particularmente aquellos que cuentan con la validación internacional o certificación. Esta revisión se adelantó mediante consultas por internet y, en la medida de lo posible, mediante consulta directa con las instituciones.

Se utilizó una matriz para estudiar la estructura de los SGA consultados, en la cual se tabuló el índice de contenidos del documento maestro de los SGA, y se compararon y analizaron los elementos comunes y diferenciadores.

A su vez, los datos de la comparación de los SGA fueron contrastados con los contenidos establecidos en las normas ISO 14001 para determinar los puntos congruentes y elementos disímiles entre los SGA y el contenido de la norma.

\section{SISTEMA DE GESTIÓN AMBIENTAL}

Existen varias definiciones de SGA. Para el caso de los SGA de interés para las universidades y entidades académicas, es conveniente partir de la definición de Ryding ${ }^{2}$ por la connotación integradora que establece: "el conjunto de disposiciones y actuaciones necesarias que permitan lograr el mantenimiento de un capital medioambiental suficiente para que la calidad de vida de las personas y el patrimonio natural sean lo más elevados posible, todo ello dentro del complejo sistema de relaciones económicas y sociales que condicionan ese objetivo".

Dicho en otras palabras, un SGA es una estructura regulatoria que surge dentro de una organización para la definición de políticas, planeación e implementación de tareas que conservan, mejoran y protegen el medio ambiente, y que da como resultados beneficios tanto dentro como fuera de la institución.

El SGA que nos interesa, parte de la gestión empresarial bajo una concepción sistémica, basada en la descentralización empresarial, donde tiene una gran relevancia la participación y capacitación profesional en el marco de la planificación estratégica de la organización. Estos sistemas tienen funciones estrechas con la gestión empresarial propiamente dicha, donde la orientación o misión de la empresa determina el enfoque del sistema y se entrecruza con los procesos de planificación estratégica y control de gestión y operación de la misma.
Un SGA es una estructura regulatoria que surge dentro de una organización para la definición de políticas, planeacióne implementación de tareas que conservan, mejoran y protegen el medio ambiente. 
A partir de los años 90 los institutos de normalización y estandarización nacionales emprendieron proyectos para desarrollar las normas de certificación de la gestión y las auditorias medioambientales.
De acuerdo con varios autores "la planificación estratégica es el proceso de formulación y revisión de estrategias que permitan el cumplimiento adecuado de los objetivos generados a partir de la política general de una organización"3. Este proceso, bajo la visión sistémica, conlleva a aplicar controles de gestión y operación, lo cual significa observar en la realidad la forma como se adapta y modifica lo planificado para poder corregir o cambiar la trayectoria propuesta. Tales procesos de control o seguimiento incluyen el análisis de los recursos y el control de la información para poder mantener bajo observación el proceso operativo; estos a su vez son procesos que retroalimentan la gestión de la organización.

En este marco se desarrollan los SGA cuyo objetivo se centra en comprobar el cumplimiento de la normativa medioambiental y definir las responsabilidades sociales y económicas de la organización. En el cumplimiento de este amplio objetivo, las organizaciones privadas y públicas de todos los sectores han emprendido diferentes caminos para establecer y poner en practica una gestión responsable y ambientalmente sostenible. Desde los años setenta, países como Estados Unidos, Holanda, Reino Unido vienen desarrollando sus propias metodologías, conocidas inicialmente como "auditorias ambientales", para afrontar el reto del manejo sostenible y a partir de los años 90 los institutos de normalización y estandarización nacionales emprendieron proyectos para desarrollar las normas de certificación de la gestión y las auditorias medioambientales.
Actualmente, el modelo más generalizado para SGA es la norma ISO 14001, que estandariza las normas desarrolladas en los años 90 aprobada en 1996 y que se convirtió en muy poco tiempo en la norma con mayor trascendencia global por sus implicaciones dentro de la cultura organizacional en la industria de todo el mundo. La norma ISO 14001 busca armonizar el funcionamiento de las organizaciones alrededor del globo con su comportamiento ambiental. ISO 14000 es el nombre genérico de las normas para la gestión ambiental, pero es ISO 14001 la norma que mide la conformidad del SGA de una organización con los requisitos específicos y es la única norma de la serie ISO 14000 en la que una organización busca certificarse. El objetivo de las normas ISO 14000 es dar a una organización un esquema para manejar sus aspectos ambientales.

Su diseño parte del análisis de normas previas existentes, de gran aceptación y eficacia demostrada; como la norma británica BS 7750 y las normas de la comunidad europea EMAS (Eco-Management and Audit Scheme) de las cuales fueron asimiladas su estructura, principios funcionales y procedimientos de verificación de prácticas de gestión ambiental por terceras partes, tendientes a garantizar la congruencia de las prácticas de control con los objetivos y metas establecidas por la organización.

El carácter genérico de la norma hace que ésta pueda ser adoptada por cualquier tipo de organización, de cualquier tamaño en cualquier parte del mundo; obedeciendo con esto a las demandas de una población global cada vez más sensible a los cambios del medio ambiente y deseosa de organiza- 
ciones cuyos productos y servicios ofrezcan garantías con respecto a su compromiso con la preservación del bienestar ambiental global.

La gestión ambiental tal como fue concebida por ISO, no se limita al manejo adecuado de residuos o a la prevención de impactos negativos que puedan ocasionar el proceso productivo o administrativo de una empresa en particular y extiende sus alcances hacia la relación con el usuario final de los productos y/o servicios de una organización; incluso se ha involucrado a la comunidad en general. Al presente se maneja la versión ISO 14001:2004.

\subsection{IMPLEMENTACIÓN de UN SISTEMA DE GESTIÓN AMBIENTAL}

En particular un sistema de gestión ambiental contiene cuatro partes fundamentales: la política y la planificación, la operación, el seguimiento y el mejoramiento del desempeño ambiental.

Para iniciar el proceso de implementación del sistema de gestión, la dirección de la organización debe identificar y asignar los recursos adecuados y crear una estructura de personal para planificar y dirigir el proceso de implementación.

El representante de la dirección será el encargado de mantener el funcionamiento del SGA e informar a los demás miembros de nivel superior acerca de los avances en la implementación, identificar recursos y personal disponible, desarrollar procedimientos y seguir la pista de los costos y beneficios derivados del SGA.

La implementación del SGA demanda capacitación a todos los miembros de la organización acerca de los efectos que tiene el trabajo que desarrollan sobre el medio ambiente.
La norma ISO 14001 requiere documentación. Básicamente, solicita que la organización documente -mediante procedimientos, manuales, instrucciones de trabajo, procesos y otros registros- una imagen clara del SGA de su instalación y el grado de afinidad de éste con los planteamientos de la norma ISO 14001. La documentación es crítica ya que ofrece evidencias objetivas sobre el estado del SGA de la organización. Esta documentación debe estar almacenada en un archivo que contenga además registros sobre permisos de autoridades ambientales, control ambiental, calibración de equipos y otros registros que proporcionen evidencia del estado del SGA.

\subsection{ReQUISITOS DEL SISTEMAISO 14000}

Los requisitos para establecer un SGA son:

1. Definir la política ambiental apropiada a la naturaleza del negocio, que sirve como referente para la definición de los objetivos y metas ambientales de la organización disponible al público.

2. Planificar estableciendo y manteniendo procedimientos para la identificación de aspectos ambientales de las actividades, productos y servicios de la organización que puedan ser controlados o causar un impacto importante al medio ambiente.

3. Definir los objetivos y metas ambientales documentadas en cada nivel de la organización, objetivos consistentes con la política de calidad y los compromisos de minimización de la contaminación.

4. Establecer los programas de gestión ambiental para garantizar el cumplimiento de los objetivos y metas ambientales de la organización, designando funciones, responsabilidades específicas para cada nivel de la organización, y
Un sistema degestión ambiental contiene cuatro partes fundamentales: la política y la planificación, la operación, el seguimiento yel mejoramiento del desempeño ambiental. 
definir un tiempo y recursos necesarios para la consecución de cada objetivo.

5. Una vez definidos los programas de gestión ambiental, se implementan y operan conforme con la estructura y responsabilidades definidas. Esto incluye definir los procedimientos para la comunicación y difusión del SGA, la documentación del sistema y control de documentos.

6. Identificar las acciones asociadas con impactos ambientales para asegurar que se lleven a cabo bajo condiciones especificas, es decir, ejercer el control operativo estableciendo y manteniendo procedimientos específicos, detallando criterios operativos, incluso compras y contrataciones, y preparando las actividades y programas de emergencia.

7. Una vez puesto en marcha el SGA es necesario generar los procedimientos para controlar y medir cualquier desviación de los objetivos y metas de la organización, no sólo de las operaciones y actividades, sino también del funcionamiento de los equipos. Este proceso permite evaluar periódicamente el cumplimiento de las regulaciones ambientales que afectan a la organización.

8. Cuando haya lugar, es necesario tomar acciones correctivas y preventivas definidas previamente. Las acciones correctivas y preventivas deben ser apropiadas a la magnitud del problema y a su impacto ambiental. Cualquier cambio implementado como resultado de las acciones correctivas o preventivas debe ser registrado en los procedimientos documentados.

9. Establecer y mantener procedimientos para la identificación, mantenimiento y eliminación de los registros ambientales (incluyendo registros de capacitación, resultados de auditorias y revisiones).

10. Hacer auditorias periódicas del SGA para determinar si está o no conforme con los requerimientos de la norma y si ha sido o no debidamente implementado y mantenido. Los resultados de la auditoria deben ser comunicados a la dirección para revisiones periódicas.

11. Finalmente, es necesario que la alta dirección revise el SGA para garantizar su eficacia, con el fin de identificar las posibles oportunidades de mejora continua del sistema en sus políticas, objetivos y metas de la organización.

\section{SISTEMAS DE GESTIÓN AMBIENTAL EN UNIVERSIDADES}

Las universidades introdujeron aspectos medioambientales en su esquema de gestión a partir de los años sesenta. Las experiencias iniciales surgieron en los Estados Unidos, simultáneamente con las primeras promociones de profesionales en ciencias ambientales, las que se extendieron a lo largo de los años setenta; y en los años ochenta empiezan a desarrollarse políticas más específicas como la gestión de residuos y la eficiencia energética. Durante la década de los noventa se desarrollan políticas ambientales de gestión global, que abarcan todos los ámbitos de la institución, como puede ser el Campus Ecology de la University of Wisconsin at Madison ${ }^{4}$ o el Brown is Green, de la Universidad de Brown ${ }^{5}$.

Esta dinámica de gestión ambiental

4 Ver: http://www.fpm.wisc.edu/campusecology/

5 Ver: University of Brown. "Brown is green. Introduction and program information" <http://www.brown. edu/Departments/Brown_Is_Green/> (Consulta: 10 junio, 2003). 
en las universidades dio lugar a la creación de la Organización Internacional de Universidades por el Desarrollo Sostenible y el Medio Ambiente, Oiudsma ${ }^{6}$, que se constituyó en San José de Costa Rica en 1995. Esta organización, que actúa como una red de instituciones universitarias, tiene como objetivo el desarrollo de programas y de docentes e investigadores en el campo del medio ambiente y del desarrollo sostenible.

La universidad de Harvard en Massachussets $^{7}$, es de las primeras universidades en involucrar los impactos ambientales en la política de la institución. Otras instituciones han desarrollado campañas de sensibilización ambiental dirigidas tanto a los alumnos como al personal académico, administrativo y de servicios. Es el caso de las Universidades Politécnica de Cataluña ${ }^{8}$, cuya campaña se enmarca en su Plan de Medio Ambiente, o la Universidad de Michigan ${ }^{9}$, con la campaña Waste Management and Recycling

El proyecto Ecocampus de la Universidad Autónoma de Madrid ${ }^{10}$, además de trabajar con la comunicación y sensibilización ambiental, ha promovido la participación activa de toda la comunidad universitaria en distintos proyectos medioambienta- les y el control de la calidad ambiental del campus universitario.

Recientemente las experiencias de gestión ambiental se han extendido a distintas instituciones universitarias con el desarrollo de planes de acción que se controlan y evalúan mediante un sistema de indicadores que abarcan los distintos ámbitos de la institución. Dentro de ellas se destaca la Agenda Ambiental de la Universidad de Edimburgo ${ }^{11}$, Ecocampus de la Universidad de Tampere Yliopisto ${ }^{12}$ en Finlandia, la Oficina Verde de la Universidad Politécnica de Valencia ${ }^{13}$ y el Plan de Medio Ambiente de la Universidad Politécnica de Cataluña ${ }^{14}$.

A la fecha, se calcula que cerca de 140 universidades han incorporado políticas ambientales para la administración y gestión académica. Dentro de las universidades que han adoptado compromisos o políticas ambientales para el desarrollo sostenible, diez han sido certificados con ISO 14001, como es el caso de la Universidad de la Organización de las Naciones Unidas en Tokio.

Recientemente, algunas universidades españolas han emprendido procesos para certificar sus programas de gestión

6 Web oficial de la Asociación Internacional de Universidades por el desarrollo sostenible y el medio ambiente. OIUDSMA. 2002. <http://www.ugr.es/ oiudsma/Welcome.htm> (Consulta: 10 junio, 2003).

7 Harvard University. Center for the environment gateway to Harvard University's environmental education research and outreach enterprise. $<$ http://environment.harvard.edu > (Consulta: 10 junio 2003).

8 Universidad Politécnica de Cataluña. Fem un gest d'estalvi. <http://www.upc.es/campus/energia/>. (Consulta: 10 junio, 2003).

9 University of Michigan. Waste Management Services. 2003. <http://www.plant.bf.umich.edu/grounds/ recycle/ (Consulta: 10 junio, 2003)

10 Universidad Autónoma de Madrid. Oficina Ecocampus. Ambientación de la UAM. <http://www.uam.es/ servicios/ecocampus/especifica/> (Consulta: 10 Junio, 2003).

11 University of Edinburgh. The agenda for the environment. The University of Edinburgh environmental policy statement 1990. <http://www.cecs.ed.ac.uk/greeninfo/>. (Consulta: 10 Junio, 2003).

12 University of Tampere. Ecocampus at the University of Tampere. Ecocampus project. 2001. <http://www. uta.fi/projektit/ekokampus/> (Consulta: 10 Junio, 2003).

13 Universidad de Valencia. Oficina Verde. <http://www.upv.es/ofiverde/>. (Consulta: 6 Junio, 2003).

14 Universidad Politécnica de Cataluña. 2n pla de Medi Ambient (2002) http://www.upc.edu/es/ 
ambiental. Es el caso de la Universidad de Granada que ha implantado en todos sus centros e instalaciones, un SGA, aplicando las directrices marcadas por la norma ENUNE ISO 14001, así como un plan de minimización de residuos con el fin de reducir el impacto que la labor docente, investigadora $\mathrm{y}$ administrativa produce sobre el medio ambiente.

En América Latina uno de los ejemplos destacables es el Programa Universitario de Medio Ambiente -PumA ${ }^{15}$ - de la Universidad Nacional Autónoma de México, UnAm, cuya misión es apoyar, promover, coordinar e impulsar actividades orientadas a investigar y a difundir una cultura en torno al medio ambiente. El Puma atiende la formación de recursos humanos en el área ambiental, la cual, por su carácter multidisciplinario, no encuentra cabida en las facultades y escuelas tradicionales; difunde el conocimiento mediante el ofrecimiento de cursos de actualización, y la publicación de libros sobre el tema. Igualmente, busca vincular distintos sectores de la sociedad con la UNAM y entre sí.

En Colombia, la Pontificia Universidad Javeriana ${ }^{16}$ ha establecido una política ambiental coherente con su misión. Actualmente, con el propósito de iniciar el proceso de consolidación de la política ambiental apoyada en un SGA, la universidad ha propuesto un proyecto de investigación, que sirve de base para la consolida- ción del sistema y, por consiguiente, para el seguimiento de la política.

La Universidad Externado de Colombia, por medio de la Facultad de Finanzas, Gobierno y Relaciones Internacionales, creó en el año 2000 el programa de reciclaje "Génesis", para el manejo responsable de los residuos sólidos.

Asimismo, otras universidades como la Nacional de Colombia realizaron entre otros estudios, un proyecto de investigación sobre las representaciones sociales y mapas mentales del campus universitario $^{17}$. La Universidad del Valle, EAfIt, y la Universidad Jorge Tadeo Lozano, adelantan diagnósticos ambientales en sus campus con distintos objetivos. Muchas iniciativas universitarias se basan en cátedras para la formación ambiental las cuales en su mayoría son de carácter electivo.

A continuación se hace un resumen de los programas de gestión ambiental de algunas de las universidades anteriormente mencionadas.

\subsection{UNIVERSIDAD DE LAS NACIONES UNIDAS - TOKYO18}

La Universidad de las Naciones Unidas (UNU), con sede en Tokio, inició su proceso de certificación ISO 14001 en mayo de 1999 y la obtuvo oficialmente en enero del 2001. La certificación fue otorgada por parte de la Asociación Japonesa de Estandarización ${ }^{19}$ al Centro de UnU, al Instituto de Estudios Avanzados y al Centro de Información

15 Universidad Autónoma de México. Presentación del programa universitario de Medio Ambiente. <http:// dragon.dgsca.unam.mx/puma/html/ presentacion.html> (Consulta: 10 Junio, 2003).

16 Pontifica Universidad Javeriana. Sistema de Gestión Ambiental. < http://www.javeriana.edu.co/ Facultades/ fear/institutos/inicio.htm> (Consulta: 10 Junio, 2003).

17 BERMÚDEZ G. Olga el al. Representaciones sociales y mapas mentales del campus universitario. Bogotá: Universidad Nacional de Colombia, noviembre, 2004.

18 Tomado de http://www.unu.edu/iso14001/UNU-initiative.html. Diferentes documentos. Fecha de consulta 6 de julio de 2004.

19 Número de registro JSAE 326. 
sobre el Medio Ambiente Mundial de Tokio. Es el primer organismo de las Naciones Unidas que recibe la certificación ISO14001 y la quinta institución académica que la obtiene en el Japón.

La aplicación de la norma ISO14001 en la UNU ha repercutido positivamente en lo que respecta a la preparación para situaciones de emergencia y las reducciones registradas en el consumo de energía y recursos, junto a economías conexas, así como a una mayor conciencia de todo el personal respecto del medio ambiente.

La política ambiental titulada "Going for Green” del Centro de UNU pretende contribuir al establecimiento de prácticas laborales y lugares de trabajo ambientalmente sostenibles en la comunidad mundial y local. La política ambiental establece cuatro objetivos para el logro del mejoramiento continuo del desempeño ambiental universitario y la prevención de la contaminación.

1) Enverdecer las prácticas laborales, es decir, cumplir con toda la legislación y regulaciones ambientales aplicables y con otros requerimientos que la UNU suscriba, incluyendo consideraciones ambientales en las prácticas de mejoramiento de la universidad, reutilizando, reduciendo, reciclando los materiales comprados, ahorrando energía y reduciendo los consumos de agua.

2) Enverdecer el sitio de trabajo, mejorando la calidad del ambiente de trabajo en los edificios en Tokio (calidad interna del aire, calidad del agua potable, desechos, iluminación, salud y seguridad, etc.).

3) Contribuir con la comunidad global, involucrándose en investigación, grupos de trabajo, transferencia de conocimiento y generación de proyectos que contribuyan a la sostenibilidad ambiental ${ }^{20}$.

4) Colaborar con la comunidad local, participando como vecino responsable en alternativas locales de mejoramiento ambiental y organizando eventos (como el Día Mundial del Medio Ambiente) para incrementar la conciencia sobre problemas ambientales.

\section{(VER GRÁFICA 1)}

Los objetivos planteados en esta política se adaptan a través de un plan que contiene objetivos y metas medibles y monitoreo, revisión, autoanálisis y análisis de desempeño. Incluye acciones correctivas y plantea un diálogo abierto sobre cómo mejorar el desempeño ambiental y el sistema de gestión ambiental de la universidad con todo el personal.

\subsection{UNIVERSIDAD DE BROWN - ESTADOS UNIDOS}

La universidad ha adoptado la iniciativa Brown Is Green para facilitar la conservación de recursos, estrategias de reducción de residuos e incrementar el conocimiento de los diferentes aspectos ambientales del campus.

La Universidad de Brown, desde 1990, se comprometió a promover el manejo ambiental de su campus y estableció una política ambiental para la conservación de los recursos ambientales. Esta política
La política ambiental titulada "Going for Green" del Centro de UNU pretende contribuir al establecimiento de prácticas laboralesy lugares de trabajo ambientalmente sostenibles en la comunidad mundialy local.

20. En el año 2000 coordinaba alrededor de 30 proyectos relacionados con el medio ambiente mediante una red mundial de centros de investigación y capacitación, en particular trabajos sobre el cambio climático y la ordenación del medio ambiente mundial, la explotación de la tierra y los recursos naturales y la conservación del agua, así como el desarrollo urbano e industrial sostenible. 
(GRÁFICA 1)

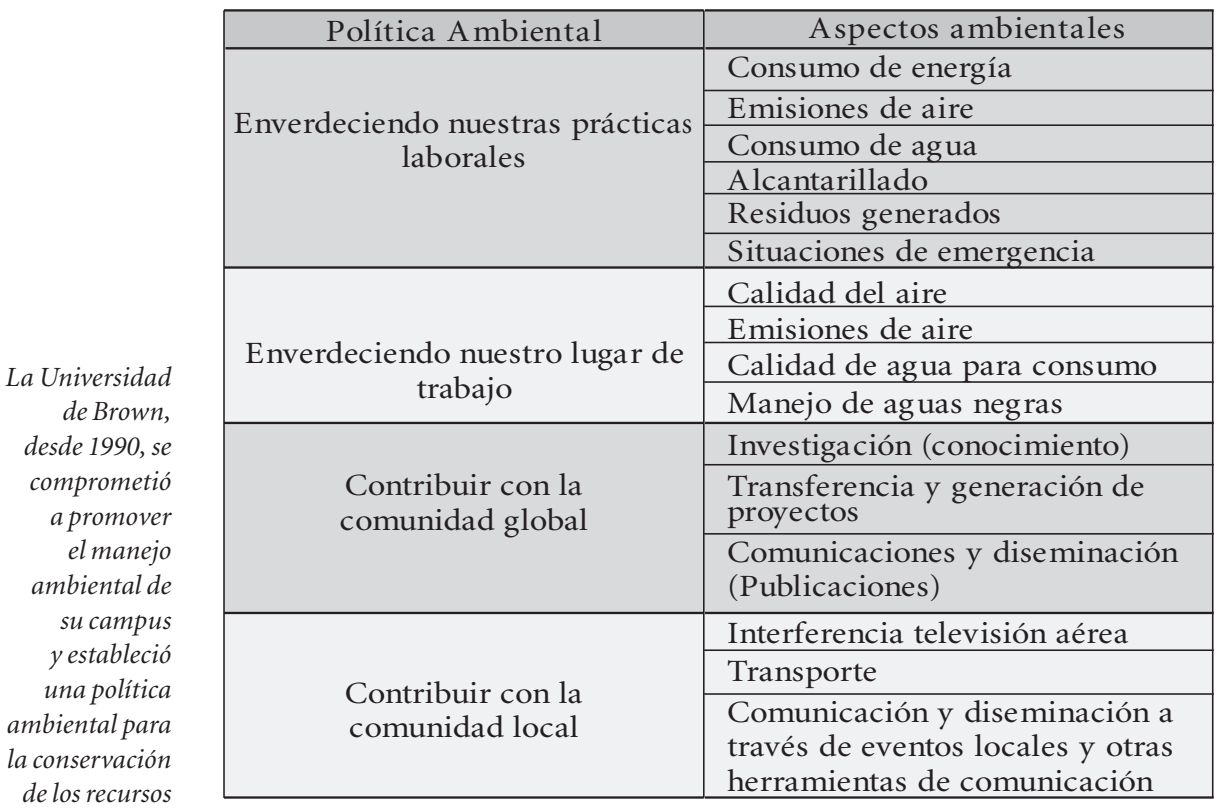

ambientales.

Estapolitica se

llama Brown

Is Green y es

un esfuerzo

cooperativo

entre todos los

departamentos

de la

universidad. se llama Brown Is Green y es un esfuerzo cooperativo entre todos los departamentos de la universidad, el profesorado, los empleados, los estudiantes y las facultades.

Brown Is Green es un programa educativo y de apoyo a partir de la alianza entre los esfuerzos de investigación y educación de los estudiantes con las oficinas administrativas. Punto fundamental para desplegar los programas para reducir los riesgos ambientales del funcionamiento de la universidad.

Brown is Green tiene los siguientes programas:

1. Programa de eficiencia energética. Busca alternativas de diseño y uso de energía con bajo consumo para áreas como control de microclima, iluminación y transporte, en conjunto con centros de investigación especializados estatales y propios relacionados con las facultades de ciencias e ingeniería.
2. Diseño de edificios. Su objetivo es el diseño, construcción y renovación de estructuras y edificios de arquitectura ambientalmente responsable y busca minimizar el impacto ambiental en los procesos de construcción y durante el ciclo de vida de dichas obras. Las actividades incluyen el diseño e implementación de estrategias para el ahorro de energía y el uso de materiales no contaminantes. Se cuenta para ello con bases de datos sobre diseño verde, nuevos materiales, sistemas alternativos para el control de microclimas y sistemas de diseño para iluminación de interiores.

3. Recuperación de recursos (reciclaje). En conjunto con el Consejo Nacional para el Reciclaje, se diseñan campañas de capacitación y recolección de artículos reciclables en los diferentes espacios del campus, así como un sistema para el reciclaje de orgánicos dentro de la organización. 
4. Programa de conservación de agua. Dentro de este programa son identificables los sistemas de flujo controlado de agua, el rediseño de sistemas de calefacción y enfriamiento para bajos consumos, además de las actividades rutinarias de auditoria para detectar oportunamente desviaciones en los programas establecidos. Los estudiantes en asocio con autoridades locales, investigan oportunidades para dar una solución más ambiental al manejo de aguas negras. Los estudios preliminares sugieren ahorros potenciales de aproximadamente 120 millones de galones de agua anualmente y ahorros por cerca de $\$ 300.000$ dólares.

5. Programa de transportes. Junto con las autoridades se han desarrollado programas para evitar el uso individual de vehículos buscando así disminuir la presión sobre áreas de parqueo, la contaminación ambiental y la congestión de tráfico en el campus. Para lograr esto se han desarrollado rutas de tránsito eficiente dentro del campus, sistemas internos de transporte masivo y uso eficiente de sistemas alternativos de combustible. Partiendo de mediciones sobre el recorrido efectuado por los miembros de la institución, se determina el nivel de uso de combustibles por persona por vehículo y el nivel de emisiones.

6. Paisaje y comidas. Existe una base de datos sobre los programas universitarios de agricultura orgánica.

7. Calidad ambiental interna y salud. Este programa tiene como principal actividad la compilación de los requerimientos por cumplir dentro del campus para garantizar el bienestar ambiental interno, seguridad y salud ocupacional. Está relacionado con las actividades del Curso 55, en el que se imparte información sobre principios básicos de toxicología, carcinogénesis, epidemiología, salud pública, percepción, medición y manejo de riesgos, además de investigaciones sobre tabaquismo y calidad ambiental en ambientes cerrados.

8. Curso de actividad ambiental institucional. En este curso que se ofrece como materia lectiva, se brinda información y capacitación sobre producción y consumo de alimentos, eficiencia eléctrica y calentamiento global, compostaje, construcción, energía y diseño, calidad ambiental de espacios interiores, sistemas de transporte, energías alternativas, calidad de agua y conservación, reciclaje y manejo de desechos.

\subsection{UNIVERSIDAD DE TAMPERE - FINLANDIA}

El proyecto Ecocampus se inició en la Universidad de Tampere en 1996. La idea del proyecto se gestó en 1995, cuando un pequeño grupo de estudiantes activos en cuestiones ambientales empezaron un plan para estudiar cómo la universidad podría reducir sus impactos negativos ambientales que ocasionaba. El proyecto tomó gran relevancia en 1996 cuando el Ministerio de Educación solicitó a todas las universidades finlandesas desarrollar e implementar un plan de desarrollo sostenible.

La política ambiental de la universidad establece principios de desarrollo sostenible y el plan de acción cubre todos los aspectos de la universidad: educación, investigación, administración y en concreto todos los impactos ambientales que la actividad cotidiana de la universidad causa. 
En la primera puesta del proyecto Ecocampus, los estudiantes participantes evaluaron los impactos ambientales de ocho diferentes departamentos de dicha Universidad. Esta información fue consignada en un reporte ambiental, el cual dio lugar a la política ambiental de la universidad. En 1997 cada departamento nombró una persona encargada de implementar estos principios ambientales. El mismo año se produjo la matriz de impactos.

\section{- OBJEtIVOS DE LA pOĹIIICA AMBIENTAL INSIITUCIONAL}

La Universidad de Tampere implementa los principios de protección y desarrollo sostenible en todas las áreas de sus operaciones: en enseñanza, investigación, y en las actividades diarias de soporte y servicios. Adicionalmente, la universidad provee información sobre asuntos ambientales y sobre los resultados logrados por grupos de personal así como por sus estudiantes.

La universidad fomenta la cooperación ambiental entre sus departamentos a través de la educación y la investigación y apoya y desarrolla cooperación en investigación ambiental y educación local, regional, nacional e internacional.

Las responsabilidades y los responsables ambientales están claramente indicados dentro de la estructura organizacional de la universidad. Las responsabilidades ambientales están definidas en el sistema de aseguramiento de calidad y en otros sistemas de gestión presentes.

Dentro del los programas y para asegurar la ejecución del sistema de gestión ambiental esta universidad ofrece educación ambiental para estudiantes: La uni- versidad y sus diferentes departamentos incorporan el aspecto ambiental y los principios de desarrollo sostenible en todas sus actividades educativas tan profundamente como sea posible. El objetivo es dar a los estudiantes, al momento de su grado, por lo menos conocimientos esenciales de asuntos ambientales globales, y capacitarlos para realizar sus actividades profesionales tanto como las de tiempo libre de una manera ambientalmente responsable. En el sistema de universidad a distancia, así como el Instituto para Estudios de Extensión desarrollan programas similares.

Entrenamiento ambiental para personal: La universidad realiza entrenamiento para todos sus miembros con el objeto de que se pueda hacer control contable ambiental de todas sus actividades tanto laborales como de esparcimiento. El personal académico puede educarse con el objeto de lograr su propio conocimiento sobre asuntos ambientales, e implementar en sus clases la calidad ambiental.

\section{- objetIVOS PARA LoS SERVIIIOS de SOPORTE yoperaciones}

Adquisición de materiales y desechos: La universidad establece objetivos de reducción de consumo de materiales y de generación de desechos en todas las áreas de la organización. Los residuos resultantes son estudiados y reciclados tan efectivamente como sea posible y los materiales que producen desechos peligrosos serán sustituidos por otros materiales. La universidad se esforzará en lograr estos objetivos realizando estudios de ciclo de vida, reduciendo consumos, y procurando materiales durables, de fácil mantenimiento y reciclables. La cantidad de materiales 
y basuras deberá ser permanentemente monitoreada.

Ahorrando energía y agua: La universidad reducirá los consumos de agua y energía en todas las formas económica y técnicamente posibles, incluyendo la adaptación de modernos sistemas, nueva tecnología. Adicionalmente, la universidad aspira a influir la actitud pública y los patrones de comportamiento diarios de su población par estos fines. Los consumos de agua y energía serán también permanentemente monitoreados.

Transporte al trabajo y otras necesidades de transporte: La universidad espera operar de manera tal que los requerimientos de transporte sean realizados de manera ambiental, y mediante su reducción. La compra de unidades de transporte deberá hacerse asegurando que su impacto ambiental sea el mínimo posible durante el ciclo de vida de estas unidades.

Operación de restaurantes: la universidad propende porque la operación de los restaurantes sea proambiental en su totalidad, y que sean estos servicios proveedores de comidas saludables y ambientalmente amigables, así como sus productos orgánicos.

Las iniciativas y medidas de diferentes unidades de la universidad son monitoreadas para lograr los objetivos ambientales de la política institucional y la coordinación es crítica para evitar la duplicación de esfuerzos. Los objetivos son revisados; a intervalos regulares se especifica la metodología para hacer el seguimiento y la revisión de los objetivos del programa en asocio con la administración de la univer- sidad. En todos los departamentos y unidades de la universidad los objetivos del programa ambiental se aplican en educación, investigación y reducción de impactos ambientales de la universidad en sus actividades diarias.

\subsection{UNIVERSIDAD DE hARVARD "INICLATIVA CAMPUSVERDE(HGCI)"}

La iniciativa Harvard Green Campus es un esfuerzo de colaboración entre las facultades, administradores, personal y estudiantes para diseñar un plan para reducir el impacto ambiental de la operación del campus y promover el medio ambiente sostenible.

En 1999, el comité Harvard University Committee on the Environment (HuCE) designó un comité intra facultades para dirigir los cambios en las operaciones ambientalmente sostenibles del campus.

El objetivo principal es analizar las prácticas convencionales de la universidad, sus resultados en el consumo de recursos y generación de contaminación, en labores de diseño y construcción, construcción y renovación, transporte, procedimientos de compra, labores de mantenimiento y comportamiento de la comunidad universitaria. El objetivo es garantizar que la universidad asegure y mantenga su posición de liderazgo en el cumplimiento de sus compromisos ambientales dentro del contexto de la consecución de sus demás objetivos organizacionales.

Los programas son:

1. Fondo de préstamos ambientales ${ }^{21}$. Provee incentivos financieros para la investigación sobre prácticas de conservación efectivas y económicamente beneficiosas. El fondo se estableció en

21 Green Campus Loan Fund (GCLF). 
julio del 2001, con siete proyectos e iniciativas de diferentes departamentos y facultades.

2. Intercambio de las mejores prácticas. Foros estudiantiles en donde se exponen ideas innovadoras que ejemplifican la excelencia de la gestión ambiental en Harvard.

3. Programa de planeación y evaluación de edificaciones sostenibles en Harvard. Tiene como propósito estimular los desarrollos de construcciones óptimas, de eficiencia financiera, estéticas y ambientalmente amables.

4. Página web Harvard Green Campus. Consiste en el diseño de un portal con información para la divulgación del programa Campus Verde.

5. Inventario de gases de invernadero. Monitoreo de las emisiones de gases de tipo invernadero generadas en las actividades rutinarias de funcionamiento de la universidad.

6. Servicios de operaciones de la universidad y programa de ahorro de energía. Destinado a ser un proveedor de soluciones para el ahorro de energía.

7. Programa de mejoramiento en consumos de energía en computadores. Un programa de ahorro de energía para uso de soluciones informáticas en las facultades de ciencias y artes, y procedimientos y criterios de compra.

8. Sistema de participación de estudiantes. Programa de investigación con asistencia de profesores para desarrollo e innovación a cargo de varias facultades.

9. Iniciativa de Campus Verde de Longwood. Para el diseño de actividades de desarrollo sostenible con eficiencia en costos y calidad en programas de educación en el campus de la escuela de medicina.

\subsection{PONTIFICIA UNIVERSIDAD JAVERIANA-COLOMBIA}

La Pontificia Universidad Javeriana dentro de los planteamientos de su misión universitaria, se ha comprometido con la solución a problemas derivados del uso irracional de los recursos naturales y del medio ambiente que incluye el propio campus de la universidad en dicho propósito, dado que al confrontar las características de la vida universitaria con la misión de la universidad, las relaciones de las personas que conforman la comunidad javeriana con su entorno vital, necesitan crear relaciones sostenibles.

En el contexto de su misión y en su compromiso con el desarrollo sostenible, adopta una política ambiental en el manejo y administración de la universidad. Esta política fomenta en la comunidad universitaria una cultura ambiental responsable que se traduce en acciones coherentes dentro y fuera del campus. Para ello, fortalece su participación en el desarrollo de planes, programas y proyectos para responder a los desafíos ambientales.

Con base en esto ha diseñado el Sistema de Gestión Ambiental con el fin de definir cambios administrativos generadores de beneficios ambientales para la universidad y que, adicionalmente, reflejen un mejor desempeño económico y redunden en la creación de una cultura ambientalmente responsable traducida en acciones coherentes dentro y fuera del campus, complementando así la formación integral de la comunidad javeriana.

La estructura propuesta para el Sistema de Gestión Ambiental de la Pontificia 
Universidad Javeriana se ha basado en los planteamientos de la NTC-ISO 14001. A continuación, se presentan los componentes de la estructura que se ha propuesto.

\section{PROGRAMASY PROYECTOS}

1. Programa de educación ambiental como dimensión o formación ambiental, que hace referencia al estudio de las relaciones existentes entre la sociedad y la base ecosistémica de sustentación, considera lo ambiental como un problema inherente a las actividades productivas. Como proceso formativo, busca que las personas conozcan, comprendan y asuman sus responsabilidades con la problemática ambiental existente, tanto en la generación como en la superación, a través de la formación de actitudes, valores y conciencia al respecto.

2. Programa de residuos sólidos. Busca promover iniciativas conducentes a realizar un manejo integral de los residuos sólidos en el campus universitario. Incluye el diagnóstico de la situación actual del manejo de los residuos sólidos, realiza experiencias piloto de manejo eficiente de residuos sólidos en diversos escenarios del campus, propone alternativas de manejo de estos residuos a la dirección de recursos físicos y propende por una cultura ambiental en el manejo de los residuos sólidos por parte de la comunidad universitaria.

3. Programa de agua. Este programa pretende mejorar el uso y el manejo del recurso agua dentro de las instalaciones de la universidad. Para esto se diagnostican los problemas de degradación ambiental en lo referente a uso, manejo y aprovechamiento del agua potable, agua lluvia y aguas residuales dentro del campus de la universidad, para identificar los puntos que representen mayores niveles de desperdicio del recurso y los puntos que representen las descargas más significativas y/o contaminantes a la red de alcantarillado de la ciudad.

4. Programa de aire y ruido. El programa de aire y ruido, pretende cuantificar la emisión de contaminantes atmosféricos y niveles de presión sonora, provenientes tanto de fuentes móviles como de fuentes fijas, para, de esta manera, evaluar su efecto sobre la salud de las personas, los recursos naturales y las propiedades, $y$ proponer medidas de mitigación y control de dichas emisiones.

5. Programa de indicadores de calidad y de gestión ambiental. Consecuentemente con el trabajo que viene realizando el Ministerio del Medio Ambiente, se reconoce la necesidad de contar con indicadores de calidad ambiental, válidos y confiables, que permitan racionalizar y cualificar las decisiones que toman como entidades de gestión y planificación.

6. Programa comportamiento ambiental del espacio construido. El programa tiene como objetivo elaborar propuestas de solución de los impactos generados en el ambiente interior de las edificaciones del campus universitario.

7. Programa de energía. Este programa pretende mejorar el uso y el manejo del recurso energético dentro de las instalaciones de la Universidad Javeriana. Diagnostica los problemas ambientales en lo referente al uso, manejo y aprovechamiento de la energía eléctrica, dentro del campus de la universidad, identificando los puntos que represen-
La estructura propuesta para el Sistema de Gestión Ambiental de la Pontificia Universidad Javeriana se ha basado en los planteamientos de la NTC-ISO 14001. 
ten mayores niveles de desperdicio del recurso y los puntos que representen los consumos más significativos, valorados desde una perspectiva ambiental.

8. Programa urbano. Busca identificar las necesidades para un adecuado funcionamiento ambiental del campus en sus espacios urbanos y su debida articulación con los componentes como la cobertura vegetal, el mobiliario, la movilidad urbana, los espacios construidos, entre otros aspectos, los que hacen que intervengan tanto los planificadores y los técnicos en temas ambientales como ecólogos, ingenieros forestales, etc, que desarrollen propuestas alternativas para un adecuado uso de los recursos en ese espacio abierto.

9. Programa de zonas verdes en el campus. Busca identificar mecanismos que permitan que las zonas verdes del campus contribuyan a mejorar las condiciones de vida de los estudiantes, profesores y demás personas que lo utilizan.

\subsection{UNIVERSIDAD NACIONAL DE COLOMBIA (BOGOTÁ) 22}

La Universidad Nacional de Colombia, establece en 1999 un grupo de trabajo integrado por directivas, docentes, personal administrativo y estudiantes para desarrollar un programa de gestión ambiental en el campus, bajo el nombre UN-Ambiente.

El SGA establece elementos de planeación como son visión, misión, objetivos general y específicos; así como el delineamiento de programas o planes maestros para la gestión del espacio público, estudio de la dimensión ambiental en los contenidos curriculares, manejo de residuos espe- ciales, manejo de residuos sólidos orgánicos, manejo de residuos sólidos, manejo de residuos líquidos y gestión del agua y divulgación. El objetivo general es hacer de la Universidad Nacional, sede Bogotá, un modelo de gestión ambiental para toda la comunidad colombiana.

Los programas diseñados son:

1. Programa espacio público. Se centra en el desarrollo de directrices para la consolidación de un plan maestro que busque empatía ente la planta física, las áreas verdes y la comunidad universitaria presentes en el campus. Este incluye criterios como movilización, esparcimiento, vigencia y apropiación.

2. Programa de dimensión ambiental. Pretende la consolidación de grupos de trabajo entre la dirección académica de la sede y los directores curriculares de cada facultad. Su primer tarea consiste en un diagnóstico del grado de inclusión dentro del plan de enseñanza de cada facultad de asuntos relacionados con la dimensión ambiental. La intención es establecer un espacio permanente de discusión sobre el tema ambiental (modalidad curso conexo o cátedra) abierto a toda la comunidad universitaria.

3. Programa de manejo de residuos especiales. Apoya la definición de lineamientos y esquemas generales de la puesta en marcha, evaluación, y mejoramiento continuo del plan de manejo de residuos especiales generados en las diferentes dependencias de la Universidad. Establece rutas sanitarias

22 Universidad Nacional de Colombia. Sistema de gestión ambiental para el campus. Cartilla No. 1. Grupo UN Ambiente. Bogotá. S.f. 
y regula el uso del horno crematorio. Además se establecerán protocolos de operación para los sectores relacionados con la generación de desechos especiales así como guías para su tratamiento. Dependiendo de la capacidad de tratamiento, se considerará la posibilidad de ofrecer el servicio a fuentes externas de desechos especiales.

4. Plan de manejo de desechos orgánicos. Pretende establecer los lineamientos para la planeación, puesta en marcha, rediseño y mejora de los sistemas de tratamiento y disposición final de este tipo de desechos mediante la aplicación de compostaje y lombricultura.

5. Plan de manejo de residuos sólidos. Pretende el establecimiento de protocolos, instrucciones, articulación y evaluación de los diferentes frentes de operación sobre desechos sólidos, teniendo como prioridad el fortalecimiento de las actividades del centro de acopio de residuos sólidos.

6. Programa de manejo de residuos líquidos y gestión del agua. Busca crear protocolos para el manejo responsable de residuos líquidos y el centro de acopio respectivo.

7. Programa de divulgación. Desarrolla la consolidación de los procesos correspondientes a la implementación del sistema de gestión ambiental, mediante la socialización de esta iniciativa dentro y fuera del campus.

\section{COMPARACIÓN DE LA ESTRUCTURA DE LOS SISTEMAS DE GESTIÓN AMBIENTAL UNIVERSITARIOS RECOPILADOS}

Se presenta a continuación una matriz en la cual se compara la estructura de los sistemas de gestión ambiental recopilados que discrimina la existencia o no de los elementos básicos constitutivos de un SGA convencional regido por principios de mejora continua, tales como: declaración explícita de una política, objetivos y metas, establecimiento de planes y programas y proyectos, sistemas de comunicación (divulgación), elementos de revisión y control (programa de auditoria) y programación de revisiones de desempeño por la dirección.

Del análisis de esta matriz (Tabla 1), se desprende que:

Tabla 1. Matriz de comparación de SGA universitarios

\begin{tabular}{|c|c|c|c|c|c|c|}
\hline Característica & 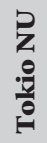 & है & 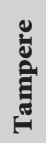 & 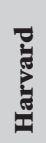 & d & 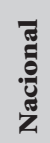 \\
\hline Política & $\mathrm{X}$ & $\mathrm{X}$ & & $\mathrm{X}$ & $\mathrm{X}$ & $\mathrm{X}$ \\
\hline Objetivos & $\mathrm{X}$ & & $\mathrm{X}$ & $\mathrm{X}$ & $\mathrm{X}$ & \\
\hline Metas & $\mathrm{X}$ & & & & & \\
\hline Planes & $\mathrm{X}$ & $\mathrm{X}$ & & $\mathrm{X}$ & & $\mathrm{X}$ \\
\hline Programas & $\mathrm{X}$ & $\mathrm{X}$ & $\mathrm{X}$ & $\mathrm{X}$ & $\mathrm{X}$ & $\mathrm{X}$ \\
\hline Proyectos de investigación & $\mathrm{X}$ & $\mathrm{X}$ & $\mathrm{X}$ & $\mathrm{X}$ & $\mathrm{X}$ & \\
\hline Comunicación & $\mathrm{x}$ & $\mathrm{X}$ & $\mathrm{X}$ & $\mathrm{X}$ & $\mathrm{x}$ & $\mathrm{X}$ \\
\hline Auditoría & $\mathrm{X}$ & $\mathrm{X}$ & $\mathrm{X}$ & $\mathrm{X}$ & & \\
\hline Revisión dirección & $\mathrm{X}$ & & & & & \\
\hline
\end{tabular}


- Los sistemas de gestión ambiental universitarios no son homogéneos en su estructura; algunos casos presentan ausencias de definiciones en puntos categóricos, como la no declaración de una política institucional o el establecimiento de objetivos y metas claras de acuerdo con lo estipulado en la norma ISO 14001.

- Es notoria la falta de definición de instrumentos de seguimiento y control (auditorias), o ausencia de prácticas que permitan rediseñar y mejorar la estructura del SGA sobre la base de mediciones y análisis de los programas establecidos en la institución (revisión por la Dirección).

- Es también notable que uno de los puntos con mayor coincidencia entre los SGA universitarios es la presencia de proyectos de investigación, que en algunos casos, por ejemplo en los de la Universidad de Harvard y la Universidad de Tampere, incluyen servicios de investigación y consultoría destinados a sectores empresariales no vinculados directamente con la universidad; así como la presencia de programas de educación específica en temáticas de sensibilización ambiental y/o de gestión ambiental dentro de la universidad, los cuales no se encuentran presentes en los sistemas de gestión ambiental empresariales, siendo bastante homogéneos en su estructura y no excluyen en sus contenidos orgánicos a ninguno de los numerales que establece la norma ISO 14001.

En la siguiente tabla tenemos una comparación de diferentes características físicas de las universidades mencionadas:

El manejo de una población fluctuante en el área del campus universita-

Tabla 2. Comparación de características físicas

\begin{tabular}{|c|c|c|c|c|c|c|}
\hline Universidad & & HARVARD & NACIONAL & JAVERIANA & TAMPERE & BROWN \\
\hline Ubicación & $\begin{array}{c}\text { TOKYO } \\
\text { (JAPÓN) }\end{array}$ & $\begin{array}{c}\text { CAMBRIDGE } \\
\text { (U.S.A.) }\end{array}$ & BOGOTÁ & BOGOTÁ & $\begin{array}{c}\text { TAMPERE } \\
\text { (SUECIA) }\end{array}$ & $\begin{array}{c}\text { RHODE } \\
\text { ISLAND } \\
\text { (U.S.A.) }\end{array}$ \\
\hline Localización & URBANA & RURAL & URBANA & URBANA & URBANA & RURAL \\
\hline Tipo & EDIFICIOS & CAMPUS & EDIFICIOS & EDIFICIOS & EDIFICIOS & CAMPUS \\
\hline Topografía & PLANA & PLANA & PLANA & LADERA & PLANA & LADERA \\
\hline Área (Hectáreas) & NA & 250 & NA & 16 & NA & NA \\
\hline Población & NA & 100.000 & NA & 30.000 & NA & 10.000 \\
\hline
\end{tabular}


rio, implica asumir un manejo más allá del mantenimiento de jardines y empradizados. En las universidades tomadas como ejemplos se nota que los programas establecidos para este fin son muchos, de diversa índole e implican una asignación presupuestal específica. Es de anotar que incluso la Pontificia Universidad Javeriana que cuenta con un campus restringido por su ubicación, tiene programas para las zonas verdes y la conservación de sus árboles. El Externado de Colombia también se presenta como ejemplo de manejo de campus universitario, ya que sus jardines son modelo de estética y belleza. De hecho esta universidad emitió una publicación sobre el tema "Bosques y Jardines del Externado”, en el año 2001.

\section{SISTEMA DE GESTÓN AMBIENTAL DEL POLITÉCNICO GRANCOLOMBIANO}

\subsection{ANTEEEDENTES}

Desde junio del 2000 el Politécnico Grancolombiano ha venido trabajando en la dimensión del concepto de "desarrollo sostenible", tomando como base la misión de la institución, en la que se expresa claramente el interés por este tema, al enunciar una educación integral e integradora basada en la generación de desarrollo sostenible.

Se decidió por lo tanto, construir una imagen conjunta sobre el papel de la universidad frente a la sostenibilidad. Esto llevó a asumir el reto de concientizar a la comunidad institucional en el sentir, pensar y actuar. Para ello propuso una transversalidad en el tema con la creación de un equipo multidisciplinario de docentes capaz de manejar los contenidos con propiedad y profundidad. Se generó entonces, una buena voluntad de trabajo y compromiso con la institución y se alcanzó el segundo paso, cuyo objetivo propuesto fue la presentación de un proyecto institucional de desarrollo sostenible dirigido a complementar los programas académicos de una manera más integral para ofrecer profesionales formados con visión frente a la sostenibilidad, concienciación y capacitación de los docentes en el tema, así como para apoyar a la institución y a las empresas en la búsqueda de la ecoeficiencia.

Por consiguiente, en lo institucional se inició la puesta en práctica de las estrategias ambientales institucionales en busca de una mejor imagen interna y externa, enfocada a la conservación del entorno natural de la universidad, las relaciones con la comunidad, y contribuir en la disminución de costos con el desarrollo de estrategias de calidad, ahorro en el consumo de recursos y optimización de procesos.

Dentro de este contexto, se hace evidente la necesidad de analizar la estructura de los SGA en diferentes universidades, con el propósito de establecer un diseño específico que facilite el cumplimiento de los compromisos ambientales del Politécnico Grancolombiano.

\subsection{DIAgNóstICO}

En la búsqueda de la consolidación del proceso se decidió que el Politécnico debía contar con un SGA que cumpliera con las NTC ISO 14001, orientado a cumplir el compromiso de garantizar el logro de la política ambiental que se adopte.

Para cumplir con este propósito, se realizaron dos investigaciones iniciales, las cuales hacen parte de una primera etapa 
de análisis de la situación ambiental de la Institución.

La primera fue el diagnóstico ambiental de la universidad siguiendo la norma ISO $14000^{23}$. En este estudio, se midieron los impactos que sobre el agua, el suelo y el aire genera la universidad y se analizó el consumo de recursos naturales, teniendo en cuenta la evolución del gasto de agua y energía desde 1998. También se analizó la situación del Politécnico frente a la legislación ambiental vigente. Esta investigación incluye la propuesta de una política ambiental institucional para ser revisada y aprobada por las directivas.

Como proyecto complementario, se efectuó la caracterización de los residuos sólidos ${ }^{24}$, por ser éste uno de los temas más relevantes dentro de los resultados arrojados por los diagnósticos previos y con el fin de diseñar el plan integral de manejo de residuos.

Al mismo tiempo, se llevó a cabo el diagnóstico de la flora y de los suelos del campus universitario ${ }^{25}$, asimismo se diseñó un plan de mejoramiento ambiental y se desarrolló un estudio general sobre la zona donde se encuentra la universidad. Además, se realizó un inventario forestal y una delimitación de áreas con la caracterización de las diferentes especies vegetales. Los datos obtenidos sirvieron para diseñar una serie de acciones dirigidas a mejorar el campus universitario, en busca de contribuir a la preservación de los Cerros Orientales de la ciudad con programas acordes con los lineamientos establecidos por las entidades del control de esta zona de Bogotá.

Los avances logrados permitieron identificar nuevas necesidades de investigación, por las características específicas de la universidad. Entre ellas el número de estudiantes nuevos que ingresan semestralmente y que deben a su vez, integrarse al SGA ya establecido, por lo que se propuso un estudio de análisis y diseño de estrategias para el programa de comunicación, educación y sociabilización del SGA. Igualmente se presentó la necesidad de diseñar e implementar un software que incluyera las nuevas variables consideradas dentro del sistema.

El análisis de las investigaciones y del proceso de implementación del SGA unido a las funciones sustantivas de la universidad en docencia, investigación y proyección social, llevó a un estudio juicioso sobre las necesidades y las falencias de un SGA universitario. Esto generó una propuesta para establecer un SGA en el Politecnico Grancolombiano, que a su vez sirva como modelo para los centros educativos.

\subsection{ESTRUCTURA GeNERAL DEL SGA para El POLITÉCWICO GRANCOLOMBIANO}

La NTC ISO 14001 se establece como una herramienta de aplicación general para todo tipo de instituciones; por lo tanto no presenta aspectos específicos aplicables a los centros de educación superior.

23 Avendaño, Hernando, Camacho, Clemencia, Castro, Pablo. Diagnóstico Ambiental de la Institución Universitaria Politécnico Grancolombiano siguiendo la Norma ISO 14001, Politécnico Grancolombiano, octubre 2003. Bogotá, Colombia.

24 Camacho, C; Soler, H.. Análisis Preliminar y Caracterización de los Residuos Sólidos de la Institución Universitaria Politécnico Grancolombiano para el Diseño de un Plan Integral de Manejo de Residuos. 2004. Bogotá, Colombia.

25 Camacho, C; Quintero, J; Muños, A.. Diagnóstico de la Flora de la Institución Universitaria Politécnico Grancolombiano y Recomendaciones para el Plan de Manejo Ambiental. 2003. Bogotá, Colombia. 
Las diferencias y a la vez las fortalezas encontradas se basan en el espíritu de investigación, su razón de ser en la formación de profesionales, la importancia de la proyección social y finalmente la localización, teniendo en cuenta el campus universitario.

Considerando los resultados obtenidos en las investigaciones previas efectuadas en el Politécnico Grancolombiano (diagnóstico ambiental, diagnóstico de flora y suelos del campus y diagnóstico de residuos sólidos), se identificó la necesidad de fortalecer las áreas de gestión propias de un centro de educación superior que no son consideradas en la formulación genérica de la norma ISO 14000 y que no se encontraron ajustadas en los casos universitarios estudiados. Se destacan algunas particularidades como la presencia de planes o programas con énfasis en el manejo ambiental del campus, la educación ambiental, la comunicación interna y externa de la gestión ambiental y la investigación sobre asuntos relacionados con la gestión o el compromiso ambiental de las universidades.

De esta manera, además de los planes o programas convencionales de gestión de residuos sólidos, manejo eficiente de energía, gestión de emisiones atmosféricas y comunicaciones ambientales, se incluye un programa relacionado con la actividad investigativa de la institución como elemento de generación de conocimiento de carácter transversal a las disciplinas presentes en la universidad; un programa de educación como complemento a la actividad anterior en el cual se incorporan contenidos ambientales a los programas de las diferentes facultades; un programa de diseño específico de comunicaciones tendiente a difundir los resultados y compro- misos institucionales alrededor de la gestión ambiental; y un programa de gestión de campus dadas las características particulares de los espacios universitarios.

Por esto la propuesta de SGA para el Politecnico Grancolombiano, basado en el esquema de la norma ISO 14001, tiene elementos que lo tipifican y dan un diseño específico para un centro de educación superior. Incluye proyectos de investigación aplicada a la misma institución, la inserción del manejo del campus y la continua formación de estudiantes en la vivencia de una herramienta ambiental aplicada, con el fin de lograr una mayor coherencia entre los principios académicos y la administración misma de la institución dentro del SGA.

El propósito es lograr un SGA específico, aplicado a la Institución, en el que se establezcan acciones que potencialicen, con un enfoque de sostenibilidad, los aspectos positivos y minimicen los aspectos negativos identificados.

\section{CONCLUSIONES}

Dada la naturaleza investigativa de la universidad, la implementación y el seguimiento de un SGA tenderá a ser modificado y a la mejora continua del sistema.

Desarrollar investigación ambiental, aplicada al campus universitario, se convierte en todo un reto al integrar el aspecto académico con las diferentes instancias institucionales frente a la responsabilidad con el medio que rodea al Politécnico Grancolombiano.

Por esto, al impulsar una serie de estudios para implementar el SGA, ajustado a la naturaleza de la organización como centro de formación académica, la universidad se enfrenta al compromiso de proyectar
Desarrollar investigación ambiental, aplicada al campus universitario, se convierte en todo un reto al integrar el aspecto académico con las diferentes instancias institucionales frente a la responsabilidad con el medio que rodea al Politécnico Grancolombiano. 
su imagen como una institución responsable y líder en el desarrollo sostenible en Colombia.

El Politécnico Grancolombiano tiene en su misión un compromiso con sus egresados en una formación que contenga la visión de sostenibilidad. Esto implica la adhesión a principios profundos que llevan a la modificación del comportamiento, por lo tanto se busca educar en una ética ambiental. Esta nueva dimensión debe ser asumida en los planes de estudio como tema transversal en las diferentes disciplinas profesionales y desarrollada dentro de la actividad académica de una manera dinámica, de acuerdo con los esquemas actuales del conocimiento. Es por esto, que poner en marcha el SGA debe estar inmerso en los procesos educativos, con un alto componente pedagógico.

Por otra parte el campus universitario es de vital importancia para cualquier institución de educación, ya que representa un aparte fundamental de su entorno. Los estudiantes pasan una gran parte de su tiempo allí de tal manera que se apropian del sitio y este se convierte en parte de su vivencia estudiantil. Por esto el manejo y la integración al SGA se torna en pieza fundamental para las universidades.

Se busca que el SGA defina cambios administrativos que generen beneficios ambientales para la universidad y que, adicionalmente, reflejen un mejor desempeño económico, con la creación de una cultura ambientalmente responsable, lo que se traduce en acciones coherentes dentro y fuera del campus, y complemente la formación integral de la comunidad universitaria.

Por ultimo, es de anotar que esta gran responsabilidad con el medio ambiente debe nacer desde la Rectoría, para que oriente las acciones encaminadas a unir esfuerzos por la construcción de una universidad comprometida con la preservación y el acertado manejo de los recursos naturales, así como promover la búsqueda constante de una mejor calidad de vida.

\section{BIBLIOGRAFÍA}

- AVENDAÑO, Hernando, CAMACHO, Clemencia y CASTRO, Pablo (2003). Diagnóstico ambiental de la Institución Universitaria Politécnico Grancolombiano siguiendo la norma ISO 14001. Politécnico Grancolombiano. Bogotá.

- CAMACho, C; QUintero, J; MUÑOZ (2003). Diagnóstico de la flora de la institución universitaria Politécnico Grancolombiano Y recomendaciones para el plan de manejo ambiental. Bogotá.

- CAMACHO, C. y SOLER, H. (2004). Análisis preliminar y caracterización de los residuos sólidos de la institución universitaria Politécnico Grancolombiano para el diseño de un plan integral de manejo de residuos. Bogotá .

- ASOCIACIÓN INTERNACIONAL DE UNIVERSIDADES POR EL DESARROLLO SOSTENIBLE Y EL MEDIO AMBIENTE. OIUDSMA. 2002. <http://www.ugr.es/ oiudsma/ Welcome.htm $>$ (Consulta: 10 junio, 2003).

- association of University LEADERS FOR A SUSTAINABLE FUTURE -ULFS. <http://www.ulsf. org >. Consulta: 6 julio, 2003. 
- ENVIRONMENTAL MANAGEMENT HANDBOOK. RYDING S.O. IOS Press. Ámsterdam, 1992. En: VEGA MORA, Leonel (1999). Gestión medioambiental. Bogotá: Tecer Mundo Editores.

- GREEN CAMPUS LOAN FUND (GCLF) HARVARD UNIVERSITY. Center for the environment gateway to Harvard University's environmental education research and outreach enterprise. <http://environment.harvard. edu> (Consulta: 10 junio 2003).

- ICONTEC (2004). Norma técnica colombiana NTC-ISO14001. Bogotá.

- PONTIFICA UNIVERSIDAD JAVERIANA. Sistema de Gestión Ambiental. <http://www.javeriana.edu. co/ Facultades/fear/institutos/inicio. htm> (Consulta: 10 Junio, 2003).

- UNiVERSIDAD AUTÓNOMA DE MADRID. Oficina Ecocampus. Ambientación de la UAM. <http://www. uam.es/servicios/ecocampus/especifica/> (Consulta: 10 Junio, 2003).

- UNIVERSIDAd AUTÓNOMA DE MÉXICO. Presentación del programa universitario de Medio Ambiente. $<$ http://dragon.dgsca.unam.mx/puma/ $\mathrm{html} /$ presentacion.html $>$ (Consulta: 10 Junio, 2003).

- UNIVERSIDAD DE VALENCIA. Oficina Verde. <http://www.upv.es/ofiverde/>. (Consulta: 6 Junio, 2003).
- UNIVERSIDAD POLITÉCNICA DE CATAlUÑa. Fem un gest d'estalvi. $<$ http://www.upc.es/campus/energia/>. (Consulta: 10 junio, 2003).

- UNIVERSIDAD POLITÉCNICA DE CATALUÑa. 2n pla de Medi Ambient (2002) http://www.upc.edu/es/

- UNIVERSITY OF BROWN. "Brown is green". Introduction and program information <http://www.brown. edu/Departments/Brown_Is_Green/> (Consulta: 10 junio, 2003).

- UNIVERSITY OF EDINBURGH. The agenda for the environment. The University of Edinburgh environmental policy statement 1990. <http://www. cecs.ed.ac.uk/greeninfo/>. (Consulta: 10 Junio, 2003).

- UNIVERSITY OF MICHIGAN. Waste management services. <http://www. plant.bf.umich.edu/grounds/recycle/ (Consulta: 10 junio, 2003)

- UNIVERSiTy OF TAMPERE. Ecocampus at the University of Tampere. Ecocampus project. 2001. $<$ http://www.uta.fi/projektit/ekokampus/> (Consulta: 10 Junio, 2003).

- UNIVERSITYWISCONSINMADISON http://www.fpm.wisc.edu/campusecology/ (Consulta 15 de junio, 2003).

- VEGA MORA, Leonel (1999). Gestión medioambiental. Bogotá: Tercer Mundo Editores. 